\title{
Using (S)TEM Techniques to Study Energy Related Materials at the Nanoscale
}

\author{
John C. Walmsley ${ }^{1,2}$, Per Erik Vullum ${ }^{1,2}$ and Randi Holmestad ${ }^{2}$ \\ ${ }^{1 .}$ SINTEF Materials and Chemistry, N-7465 Trondheim, Norway \\ 2. Department of Physics, Norwegian University of Science and Technology (NTNU), N-7491 \\ Trondheim, Norway
}

In most of our work on energy related materials - such as for example thin films of intermediate band solar cells, hydrogen storage materials and catalyst nanoparticles - we see that the atomic scale structures directly influence materials properties. Different transmission electron microscopy (TEM) techniques have turned out to be crucial to understand and improve the functional properties of these materials. Recent advances in instrument development has allowed for the use of aberration corrected TEM and scanning TEM (STEM) combined with simultaneous acquisition of spectroscopy data like EDS and EELS. These instrumental improvements give structural and electronic information on the atomic scale that could not be accessed by other means. This talk will give a few examples of projects going on in our research group, where studies of the materials at the nanoscale give important answers on how to proceed in developing better energy materials.

Quantum dots (QD) that create an energy band inside the band gap of a matrix material can be used to realize high efficiency, intermediate band solar cells. InAs/GaAs based QDs are grown in the Stranski-Krastanov growth mode by molecular beam epitaxy (MBE). In this growth mode, QDs form spontaneously beyond a critical thickness of the deposited InAs film due to the lattice-mismatch strain between the InAs and GaAs-based materials. Knowledge and control of the strain distribution in and around the QDs are important for the development and performance of the solar cell. A major challenge for the realization of intermediate band solar cells is the degradation of the QD structure due to the generation of misfit dislocations. Such defects are introduced as a result of residual compressive strain that accumulate as successive dot layers are grown. High resolution aberration corrected (S)TEM images show quantitatively how the lattice mismatch induced strain varies on the atomic scale and tetragonally distorts the lattice in a wide region that extends several $\mathrm{nm}$ into the GaAs spacer layer below and above the QDs. Using geometrical phase analysis, we show that all strain is elastically absorbed perpendicular to the growth direction. Parallel to the growth direction, the QDs are tetragonally strained with a c/a-ratio $>1$ in the dots and with a c/a-ratio $<1$ in the GaAs above and below the dots. As seen in Fig. 1, we also show how V-shaped dislocations originating at the QD/GaAs interface efficiently reduce most of the lattice mismatch induced strain [1].

Addition of small amounts of a transition metal, such as Ti, to the complex hydride $\mathrm{NaAlH}_{4}$ allows for reversible hydrogen storage with high volumetric and gravimetric capacity at low operating temperatures. This material system is therefore an attractive candidate for reversible hydrogen $(\mathrm{H})$ storage. The hydrogen can typically be used in combination with a proton exchange membrane fuel cell to run a car. The role of the transition metal with respect to the improved kinetics and reversibility of the system is still not thoroughly understood. We here demonstrate how TEM can be used to collect important information about the state and the role of the transition metal. This is extremely challenging as the material system is both atmosphere and electron beam sensitive. After going through $\mathrm{H}$ absorption cycles the material used for TEM analysis has to be loaded in a glove box and transferred to the microscope without being exposed to oxygen. Diffraction studies after $\mathrm{H}$ absorption reveal different Ti- 
containing phases embedded on the $\mathrm{NaAlH}_{4}$ surface. Our finding helps understanding the kinetics and cycle durability of these materials and supports the suggestion that the most rapid hydrogenation kinetics in the early $\mathrm{H}$ cycles can be attributed to the higher density of Ti atoms in the $\mathrm{NaAlH}_{4} / \mathrm{Al}_{2} \mathrm{Ti}$ interface [2].)

Supported catalyst nanoparticles are used in the processes related to energy storage and conversion. TEM provides an essential tool in the understanding and design of a wide range of these systems, including fuel cells gas-to-liquid conversion and exhaust processing. It is a challenge to understand the behavior of the catalyst during various stages of operation and obtain information about the mechanisms that cause them to deactivate. It is necessary to observe the materials in controlled and realistic conditions to achieve this and it is also important to combine the analysis with other techniques. For example, synchrotron-based techniques provide averaged in-situ data under realistic pressure and temperature conditions while TEM can provide detailed information about individual nanoparticles and the support [3]. Recent experience in these areas will be described, including the application of aberration corrected TEM [4].

\section{References:}

[1] PE Vullum, M Nord, SF Thomassen, C Boothroyd, R Holmestad, BO Fimland and TW

Reenaas, Quantitative strain analysis of InAs/GaAs quantum dot materials, submitted (2014).

[2] MP Pitt et al, Philosophical Magazine, 93 (2013), p. 1080.

[3] NE Tsakoumis et al, Catalysis Today, 205 (2013), p. 86.

[4] The authors acknowledge funding from the Research Council of Norway.
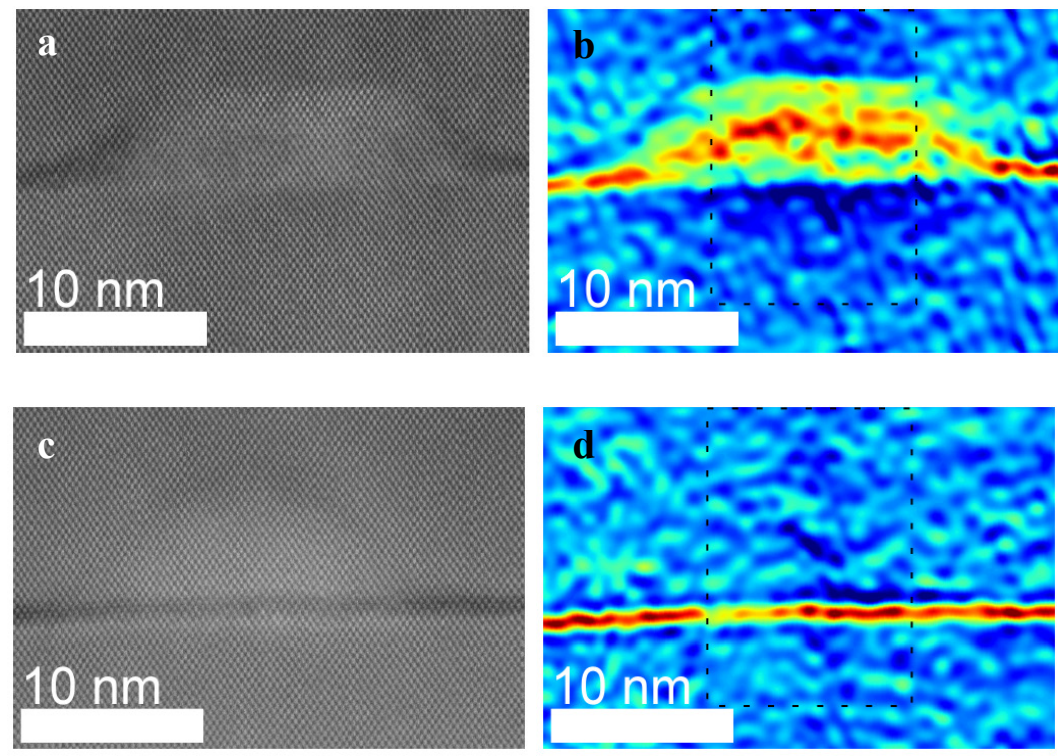

Figure 1. High resolution high angle annular dark field STEM images of InAs QDs in GaAs (a and $\mathrm{c}$ ), with corresponding strain maps for strain parallel to the crystallographic [001] direction (b and d). In c) and d) we have two dislocations in a $\mathrm{V}$ shape originating from the QD layer, relaxing the strain [1]. 